\section{Multiple myeloma}

Diana Samson FRCP FRCPath, Senior Lecturer in Haematology, Imperial College, London

Charles Singer FRCP FRCPath, Consultant Haematologist, Royal United Hospital, Bath

\section{Clin Med JRCPL 2001;1:365-70}

Multiple myeloma (MM) is a tumour of bone marrow plasma cells associated with bone pain, anaemia, infection and renal failure. A serum paraprotein is detectable in $80 \%$ of patients. Free light chains are often also detectable in urine as Bence-Jones protein (BJP), and 20\% produce only light chains (BJP MM). Rarely, serum or urine paraprotein is undetectable (non-secretory MM).

\section{Incidence and aetiology}

The annual incidence of MM is approximately 40 per million $(2,500$ new cases per year in the UK). Incidence increases with age (median 65 years), it is rare under 40, and twice as common in AfroCaribbeans. The only clearly defined epidemiological risk factor is radiation exposure ${ }^{1}$.

\section{Pathophysiology}

Immunoglobulin heavy chain $(\operatorname{IgH})$ analysis reveals that MM arises from a post-germinal centre B lymphocyte, then migrates to bone marrow where plasma cells proliferate ${ }^{2}$. Interactions with marrow stromal cells facilitate homing and growth of myeloma cells. Stromal cells produce interleukin (IL)-6 - an important growth factor for myeloma cells - which in turn produces osteoclastactivating cytokines including tumour necrosis factor $\alpha$ and interleukin (IL) $-1 \beta$ (Fig 1$)^{3}$. These cytokines upregulate stromal cell secretion of receptor activator of nuclear factor 6B ligand (RANK-L), which stimulates osteoclast production. Secretion of osteoprotogerin, the major inhibitor of RANK-L, is reduced (Fig 2). Myeloma cells also produce vascular endothelial growth factor, resulting in increased microvessel formation and tumour growth. Normal B lymphocytes are suppressed, reducing polyclonal Ig production (immuneparesis).

Complex cytogenetic abnormalities are frequent, most commonly with translocations involving chromosome 14q (site of the IgH locus) and deletions of chromosome 13 (Fig 3) ${ }^{4,5}$.

\section{Clinical features}

The commonest presenting symptom is bone pain (60\%). Bone destruction may cause hypercalcaemia. A normal serum alkaline phosphatase (SAP) reflects suppressed osteoblast activity and radionuclide bone scans are usually negative. Plain radiology may reveal osteoporosis $(5-10 \%$ of patients have only osteoporosis), lytic lesions, fractures and occasionally soft tissue extension.
Magnetic resonance imaging (MRI) is the most sensitive imaging method and is valuable in suspected cord compression where several levels may be involved. Both computed tomography and MRI are helpful in selected patients.

Renal impairment is common and usually due to tubular damage by BJP (cast nephropathy). Other causative factors include hypercalcaemia, dehydration, sepsis, glomerulonephritis, amyloidosis and non-steroidal antiinflammatory drugs. Rehydration and reversal of remediable factors should precede chemotherapy. Plasmapheresis may be helpful. The vincristine, doxorubicin, dexamethasone (VAD) regimen is well tolerated and frequently produces improvement in renal impairment. When appropriate, dialysis should accompany chemotherapy.

Approximately $15 \%$ of patients are asymptomatic and are detected as a result of an elevated erythrocyte sedimentation rate or paraprotein on screening.

Other clinical features may include anaemia, recurrent infection, hyperviscosity, lethargy and neuropathy.

Fig 1. Interaction between myeloma cells and bone marrow stroma cells (IL = interleukin; TNF = tumour necrosis factor).

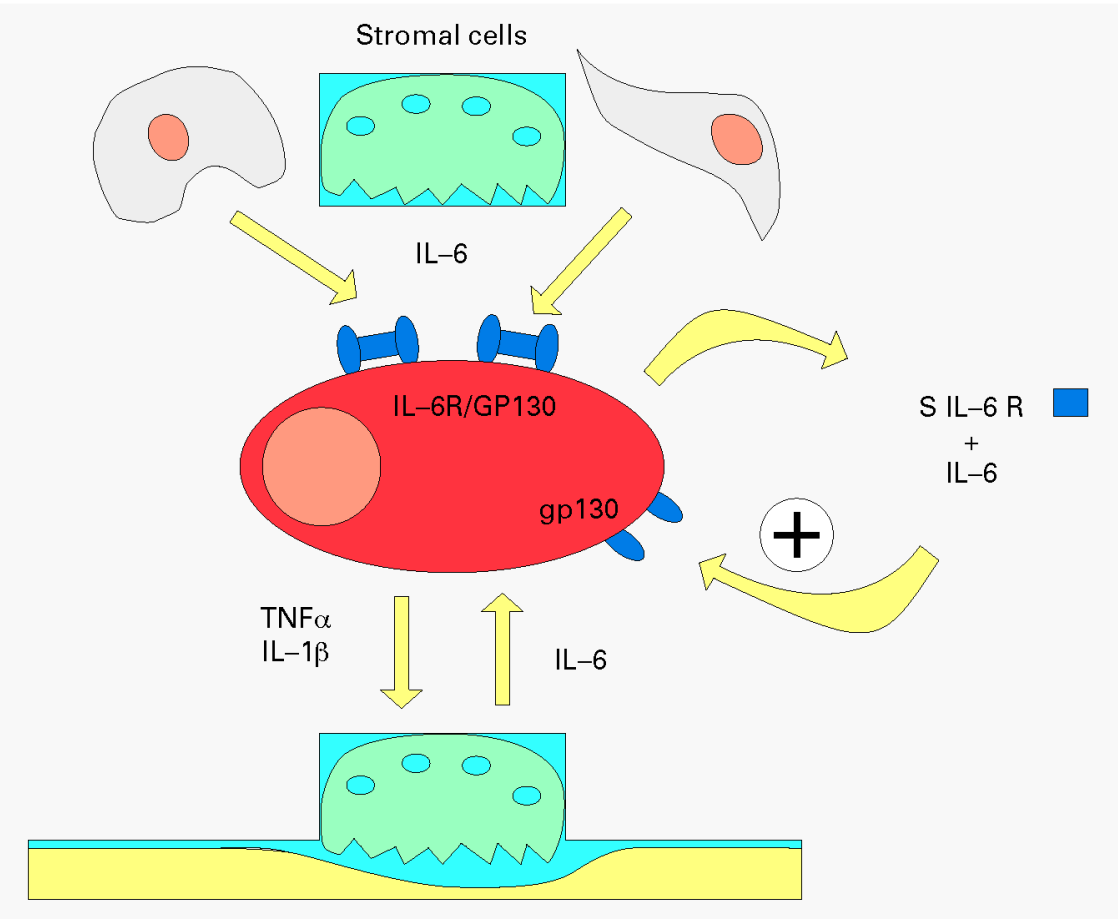




\section{CME Haematology - I}

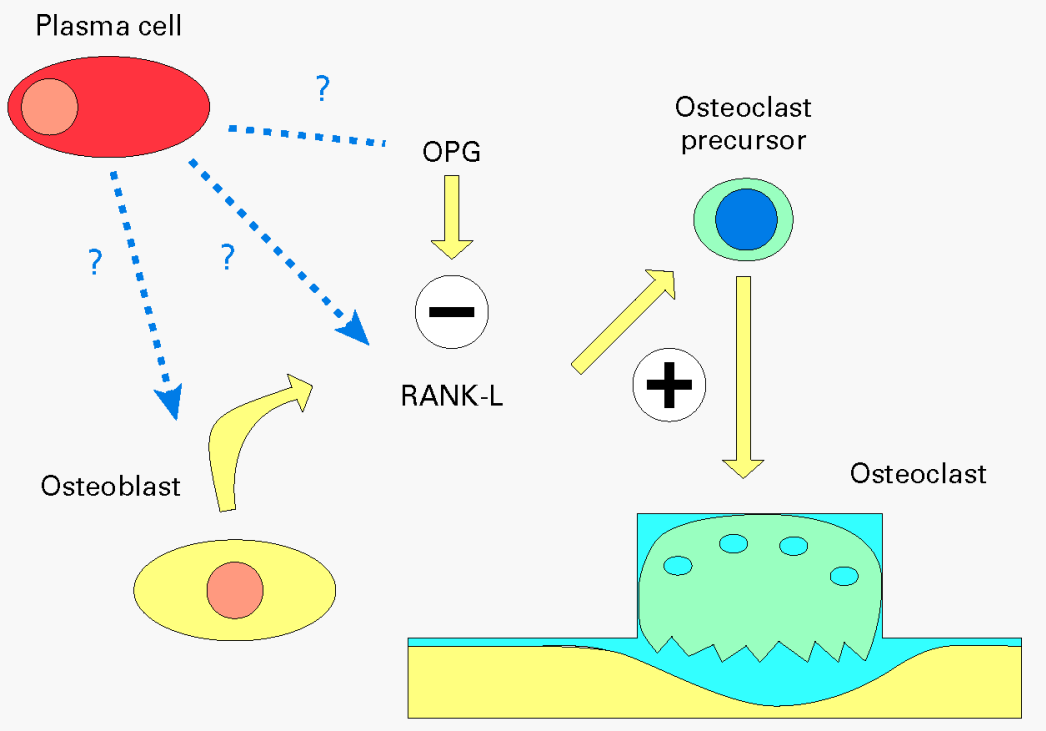

Fig 2. Osteoclast activation by myeloma cells (OPG = osteoprotogerin; RANK-L = receptor activator of nuclear factor 6B ligand).

Fig 3. Myeloma plasma cell nuclei which have been probed for two areas of $13 q 14$. Most of the cells shown are deleted for one signal of each probe, but one cell (top right) has two normal signals for both. The probes used were Rb in green (Milan YAC centre) and D13S319 in red (Vysis). Kindly donated by Dr Fiona Ross, Wessex Cytogenetics Centre.

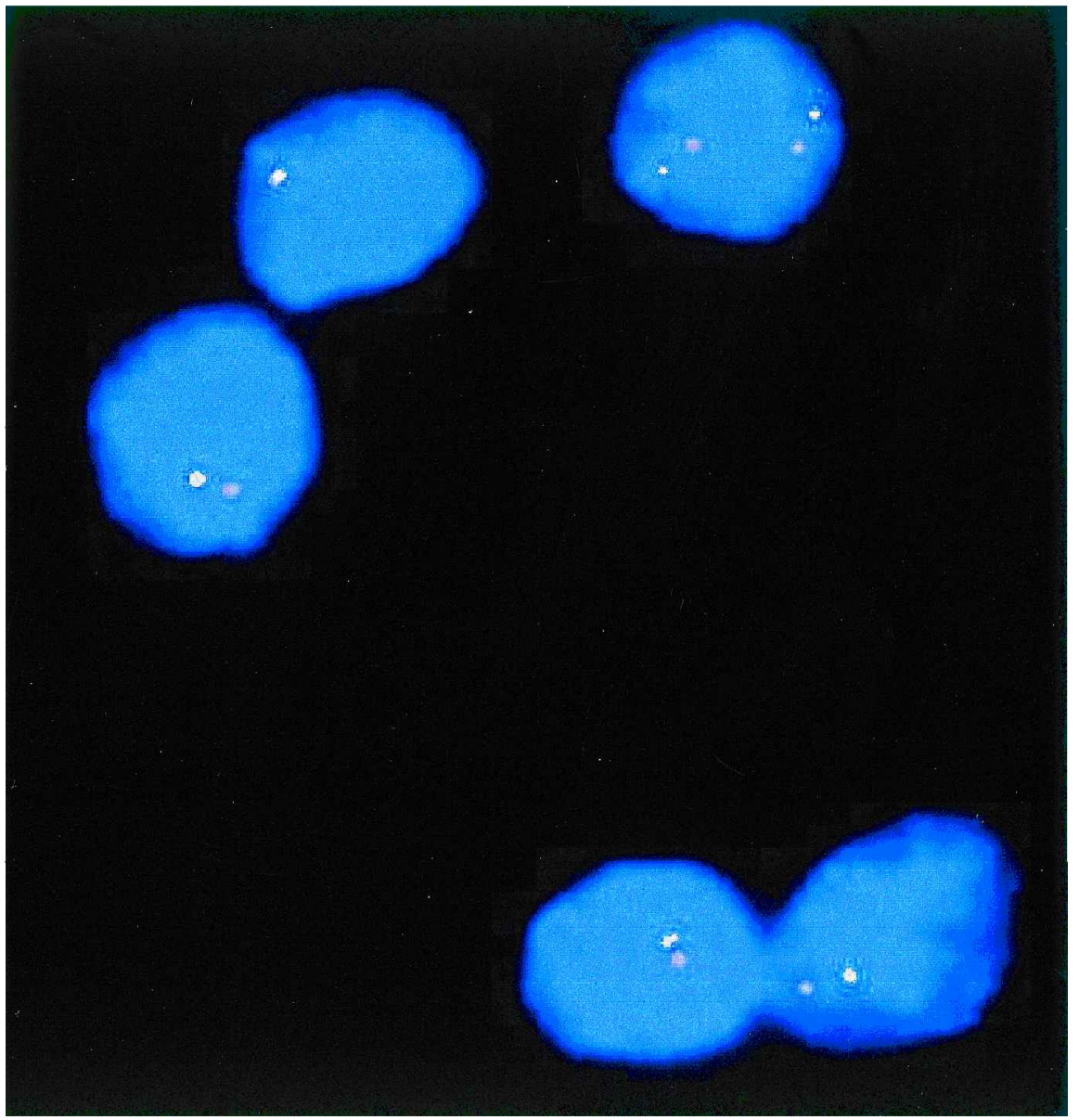

Ten per cent of patients with MM develop primary amyloid which causes nephrotic syndrome, renal and cardiac failure. Peripheral and autonomic neuropathies occur, and liver, gut and skin may be involved. The extent of amyloid deposition can be assessed using radiolabelled serum amyloid $\mathrm{P}$-component. If production of amyloidogenic protein is arrested by chemotherapy, amyloid can regress ${ }^{6}$.

\section{Differential diagnosis}

MM should be suspected particularly in patients over 50 with any of the features previously discussed. Minimal diagnostic criteria have been agreed (Table $1)^{7}$. Table 2 details useful investigations. It is important to exclude monoclonal gammopathy of undetermined significance (MGUS) and other conditions which may be associated with a paraprotein (Table 1). These include solitary plasmacytoma, primary amyloidosis and lymphoproliferative conditions (chronic lymphocytic leukaemia, non-Hodgkin's lymphoma and Waldenström's macroglobulinaemia).

\section{Monoclonal gammopathy of undetermined significance}

MGUS describes the presence of a paraprotein without evidence of MM, Waldenström's macroglobulinaemia, amyloidosis or lymphoma (Table 1$)^{8}$. There is a risk of transformation to MM (actuarial rate $16 \%$ at 10 years). The incidence of MGUS increases with age ( $3 \%$ over the age of 70 ). Patients do not have anaemia, renal failure or bone lesions. Differentiation between MGUS and early MM may be difficult, and no single test is reliable. If uncertainty persists after investigation, serial paraprotein measurements and review of clinical and laboratory parameters are necessary.

The term smouldering myeloma (SMM) identifies asymptomatic patients fulfilling diagnostic criteria for MM rather than MGUS, but without bone disease and with a stable paraprotein without treatment. Patients with MGUS or SMM should be closely monitored 
Table 1. Diagnostic criteria for myeloma and MGUS.

\section{Myeloma}

At least two out of the following three findings:

- $\quad>10 \%$ plasma cells in bone marrow or plasmacytoma on histology

- Serum paraprotein (usually $\lg G>30 \mathrm{~g} / \mathrm{l} ; \lg A>20 \mathrm{~g} / \mathrm{l}$ ) or Bence Jones proteinuria

- Osteolytic lesions on skeletal survey

\section{MGUS}

All of the following:

- no symptoms suggestive of myeloma

- $\quad$ serum paraprotein $<30 \mathrm{~g} / \mathrm{l}$

- $\quad<10 \%$ plasma cells in bone marrow

- little or no urine paraprotein (BJP)

- $\quad$ no bone lesions

- no anaemia, hypercalcaemia or renal impairment

- stable paraprotein and other parameters on observation

without treatment. Review and paraprotein requantification should occur after 3-6 months, and then annually in MGUS patients and 3-6 monthly for SMM, unless symptoms supervene.

\section{Prognostic factors}

Median survival is 3-4 years, but ranges from a few months to over 10 years. Several adverse prognostic factors have been identified, including high paraprotein levels, low $\mathrm{Hb}$, hypercalcaemia, advanced lytic bone lesions, and abnormal renal function. These factors are combined in the widely used DurieSalmon staging system ${ }^{9}$ (Table 3 ). Serum

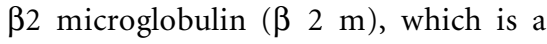
measure of tumour mass, is the most powerful prognostic factor and alone predicts survival regardless of renal function or Durie-Salmon stage. Other adverse prognostic factors are a raised CRP (a surrogate marker for raised serum IL-6) and low serum albumen. A new staging system based on $\beta 2 \mathrm{~m}$ and CRP usefully divides patients into three

Table 2. Investigation of patients with suspected myeloma.

\section{Useful screening tests}

- Full blood count and film: anaemia often present: film may show rouleaux

- $\quad$ ESR or plasma viscosity: raised in the presence of a serum paraprotein

- Urea and creatinine: may indicate renal impairment

- Calcium, phosphate, alkaline phosphatase and albumin: may reveal hypercalcaemia or low albumin

- Serum immunoglobulins: to detect immuneparesis

- Serum protein electrophoresis: to detect paraprotein

- Routine urinalysis: to detect proteinuria

- Urine electrophoresis for BJP: to detect paraprotein

- X-ray of sites of bone pain: may reveal pathological fracture or lytic lesion(s)

\section{Diagnostic Tests}

- Bone marrow aspirate: to identify plasma cell infiltration

- $\quad$ Skeletal survey: to identify lytic bone lesions not already detected

- Paraprotein typing and quantification: to characterise paraprotein

Tests to establish tumour burden and prognosis

- Serum beta 2 microglobulin: measure of tumour load

- $\quad$ Serum C reactive protein: surrogate measure of IL-6

- Serum LDH: measure of tumour aggression

- Serum albumin: when low reflects poor prognosis

- Cytogenetics: prognostic value

Tests which may be useful in some patients

- Creatinine clearance \& 24 hour proteinuria

- $\quad$ MRI: not routine but useful in patients with cord compression or solitary plasmacytoma and abnormal in $30 \%$ of patients with normal skeletal survey

- $\quad \mathrm{CT}$ : where clinically indicated

- $\quad$ Biopsy for amyloid and SAP scan: where suspected

risk groups when no other cause of raised CRP is present:

- both markers below $6 \mathrm{mg} / \mathrm{L}$ : low risk, median survival 54 months

- either elevated: intermediate risk, median survival 27 months

- both elevated: high risk, median survival six months.

Deletion of $13 \mathrm{q}$ is an important adverse prognostic factor ${ }^{11}$. Renal impairment is a risk factor only because of its association with high tumour burden $^{12}$.

\section{Initial treatment}

It is important to address general aspects of care (Table 4).

\section{Standard chemotherapy: melphalan and prednisolone}

An objective response ( $\geq 50 \%$ paraprotein reduction) is produced in $50 \%$ of patients with melphalan $6-8 \mathrm{mg} / \mathrm{m}^{2} /$ day combined with prednisolone 40-60 $\mathrm{mg}$ /day given for four days at 4-6 week intervals ${ }^{13}$. Treatment should continue until maximum response, which may take 9-12 months, and a plateau phase may be achieved. Complete response is rare. Maintenance chemotherapy in plateau phase is ineffective and adds toxicity ${ }^{14}$. Patients should be monitored to detect a rising paraprotein as progression occurs after a median time of 18 months. Those who achieve a durable plateau will often have further responses with the same chemotherapy. However, eventual resistance to melphalan is inevitable. Melphalan and prednisolone remain an appropriate treatment for older patients with $\mathrm{MM}$ as it is well tolerated.

\section{Combination chemotherapy}

Many combination regimens have been examined. The most widely used in the $\mathrm{UK}$ is doxorubicin, carmustine, cyclophosphamide and melphalan (ABCM). Only two large multicentre studies have reported survival benefit 


\section{Table 3. The Durie-Salmon staging system.}

Patients are staged as I, II or III and A or B. Stage represents tumour burden not sites involved.

Tumour cell mass

Monoclonal $\lg G(g / l)$

Monoclonal $\operatorname{lgA}(\mathrm{g} / \mathrm{l})$

BJP excretion ( $\mathrm{g} / 24 \mathrm{hr}$ )

$\mathrm{Hb}(\mathrm{g} / \mathrm{dl})$

Calcium (mmol/l)

Lytic lesions

Stage I
low
all of the
following:
$<50$
$<30$
$<4$
$>10$
$<2.6$
none or one

Stage A: Serum creatinine $>175 \mu \mathrm{mol} / \mathrm{l}$

Stage B: Serum creatinine $>175 \mu \mathrm{mol} / \mathrm{l}$

over melphalan and prednisolone ${ }^{15,16}$, though complete response is more common (but still $<10 \%$ ). A metaanalysis of 27 randomised trials also showed no survival benefit ${ }^{17}$.

The VAD regimen involves continuous intravenous infusion of vincristine and doxorubicin over four days with dexamethasone. This produces improved overall (60-80\%) and complete (10-25\%) response rates ${ }^{18}$. Although not durable, maximum response is rapidly achieved. This regimen is nontoxic to stem cells, so VAD is used in patients proceeding to stem cell harvest and autologous transplantation. The substitution by methylprednisolone of dexamethasone (VAMP) has similar effects.

\section{Table 4. General aspects of care.}

Pain control

Limitation of renal damage

Hypercalcaemia

Bone disease

Cord compression

Anaemia

Infection

Hyperviscosity syndrome

\section{Bisphosphonates}

Osteoclast bone resorption is inhibited by bisphosphonates. Patients on longterm bisphosphonates experience less bone pain and fewer fractures ${ }^{19}$. There is evidence that bisphosphonates may prolong survival ${ }^{20,21}$. Long-term bisphosphonate treatment is recommended for patients with $\mathrm{MM}^{7}$.

\section{Maintenance interferon-a}

A meta-analysis of 12 trials $^{22}$ demonstrated improved response duration (median 6 months) but no survival benefit in patients receiving maintenance interferon (IFN)- $\alpha$ during plateau phase. IFN- $\alpha$ has frequent side effects and an unfavourable cost-utility profile.

\section{High-dose therapy and stem cell transplantation}

\section{Autologous transplantation}

High-dose therapy (HDT) has been used to improve disease control and prolong survival. It usually comprises high-dose melphalan (140-200 $\mathrm{mg} / \mathrm{m}^{2}$ ) with or without total body irradiation, supported with peripheral blood progenitor cell or bone marrow transplantation after initial response to VAD. This is a safe procedure for patients under 65 years but is not curative. Median remission duration is two years. In a single prospective randomised trial which compared standard therapy with $\mathrm{HDT}^{23}$, MM patients receiving HDT had significantly better five-year survival ( $52 \%$ vs $12 \%, p=0.03)$. A case-control study has also shown a survival advantage for $\mathrm{HDT}^{24}$. HDT should form part of the primary treatment strategy in patients aged 60 years or less ${ }^{7}$.

\section{Allogeneic transplantation}

Allogeneic transplantation from a matched sibling is applicable to patients up to the age of 50 years. Transplantrelated mortality from graft versus host disease and infection is high, and relapse is also a problem. Nevertheless, donor marrow exerts a graft versus myeloma effect $^{25}$ and there is projected long-term survival of $35 \%{ }^{26}$. Allogeneic bone marrow transplantation in first remission offers $33 \%$ risk of transplant-related mortality, $33 \%$ chance of survival with recurrence, and 33\% chance of durable remission and possible cure. Lower intensity non-myeloablative conditioning regimens are being examined to exploit the graft versus myeloma effect with less toxicity.

\section{Disease progression}

Good supportive therapy is essential. Radiotherapy to sites of bone pain is usually effective palliative therapy. Oral dexamethasone may achieve further responses $^{27}$ and low-dose oral cyclophosphamide may slow progression. Thalidomide achieves responses in 30\% of chemorefractory patients ${ }^{28}$. 


\section{The future}

Recent results suggest that thalidomide and dexamethasone in combination produce responses in $70 \%$ of patients. The mode of action of thalidomide is complex, but appears to be immunomodulatory rather than antiangiogenic. Less toxic derivatives are under examination. Proteasome inhibitors affect intracellular signalling, induce apoptosis and show early promise. Immunisation strategies are also on trial.

\section{References}

1 Herrinton LJ, Weiss NS, Olshan AF. Epidemiology of myeloma. In: Malpas JS, Bergsagel DE, Kyle RA, Anderson KC (eds). Myeloma, biology and management. Oxford: Oxford University Press, 1998:150-86.

2 Van Riet I, Vanderkerken K, de Greef C, Van Camp B. Homing behaviour of the malignant cell clone in multiple myeloma. Review. Med Oncol 1998;15:154-64.

3 Croucher PI, Apperley JF. Bone disease in multiple myeloma. Review. Br J Haematol 1998;103:902-10.

4 Nishida K, Tamura A, Nakazawa N, Ueda Y, et al. The Ig heavy chain gene is frequently involved in chromosomal translocations in multiple myeloma and plasma cell leukaemia as detected by in situ hybridization. Blood 1997;90:526-34.

5 Calasanz MJ, Cigudosa JC, Odero MD, Ferreira C, et al. Cytogenetic analysis of 280 patients with multiple myeloma and related disorders: primary breakpoints and clinical disorders. Genes Chromosomes Cancer 1997; 18:84-93.

6 Gillmore JD, Hawkins PN, Pepys MB. Amyloidosis: a review of recent diagnostic and therapeutic developments. $\mathrm{Br} J$ Haematol 1997;99:245-56.

7 UK Myeloma Forum. Guidelines on the diagnosis and management of multiple myeloma. Br J Haematol 2001 (in press).

8 Kyle RA. Monoclonal gammopathy of undetermined significance and solitary plasmacytoma. Review. Hematol Oncol Clin North Am 1997;11:71-87.

9 Durie BG, Salmon SE. A clinical staging system for multiple myeloma. Correlation of measured myeloma cell mass with presenting clinical features, response to treatment, and survival. Cancer 1975;36: 842-54.

10 Bataille R, Boccadoro M, Klein B, Durie B, Pileri A. C-reactive protein and serum beta2 microglobulin produce a simple and powerful myeloma staging system. Blood 1992;80:733-7.

11 Tricot G, Barlogie B, Jagannath S, Bracy D,

Key Points

Differentiation of multiple myeloma from monoclonal gammopathy of undetermined significance can be difficult and may require a period of observation

Prognostic factors can identify patients with multiple myeloma in whom treatment may not be necessary and others who may need aggressive treatment

Renal impairment can be reversed by early chemotherapy, and dialysis is appropriate supportive therapy in some patients

Bisphosphonate therapy appears to provide a beneficial reduction in bone complications

Allogeneic bone marrow transplantation should be discussed with younger patients ( $<55$ years) with myeloma with a compatible sibling donor as this may be curative

et al. Poor prognosis in multiple myeloma is associated only with partial or complete deletion of chromosome 13 or abnormalities involving $11 \mathrm{q}$ and not with other karyotype abnormalities. Blood 1995;86: $4250-6$.

12 Iggo N. Management of renal complications. In: Malpas JS, Bergsagel DE, Kyle RA, Anderson KC (eds). Myeloma, biology and management. Oxford: Oxford University Press, 1998:381-401.

13 Alexanian R, Haut A, Khan AU, Lane M, et al. Treatment for multiple myeloma. Combination chemotherapy with different melphalan dose regimens. JAMA 1969;208:1680-5.

14 Belch A, Shelley W, Bergsagel D, Wilson K, et al. A randomized trial of maintenance versus no maintenance melphalan and prednisone in responding multiple myeloma patients. Br J Cancer 1988;57: 94-9.

15 Salmon SE, Haut A, Bonnet JD, Amare M, et al. Alternating combination chemotherapy and levamisole improves survival in multiple myeloma: a Southwest Oncology Group Study. J Clin Oncol 1983;1:453-61.

16 MacLennan IC, Chapman C, Dunn J, Kelly K. Combined chemotherapy with ABCM versus melphalan for treatment of myelomatosis. The Medical Research Council Working Party for Leukaemia in Adults. Lancet 1992;339:200-5.

17 Myeloma Trialists' Collaborative Group. Combination chemotherapy versus melphalan plus prednisone as treatment for multiple myeloma: an overview of 6,633 patients from 27 randomized trials. J Clin Oncol 1998;16:3832-42.

18 Samson D, Gaminara E, Newland A, Van de Pette $\mathrm{J}$, et al. Infusion of vincristine and doxorubicin with oral dexamethasone as first-line therapy for multiple myeloma. Lancet 1989;ii:882-5.

19 Berenson JR, Lichtenstein A, Porter L, Dimopoulos MA, et al. Efficacy of pamidronate in reducing skeletal events in patients with advanced multiple myeloma. Myeloma Aredia Study Group. N Engl J Med 1996;334:488-93.

20 Berenson JR, Lichtenstein A, Porter L, Dimopoulos MA, et al. Long-term pamidronate treatment of advanced multiple myeloma patients reduces skeletal events. Myeloma Aredia Study Group. J Clin Oncol 1998;16:593-602.

21 McCloskey EV, MacLennan IC, Drayson MT, Chapman C, et al. A randomized trial of the effect of clodronate on skeletal morbidity in multiple myeloma. MRC Working Party for Leukaemia in Adults. $\mathrm{Br} \mathrm{J}$ Haematol 1998;100:317-25.

22 The Myeloma Trialists' Collaborative Group. Interferon as therapy for multiple myeloma: an individual patient data overview of 24 randomized trials and 4012 patients. Br J Haematol 2001;113:1020-34.

23 Attal M, Harrousseau JL, Stoppa AM, Sotto $\mathrm{JJ}$, et al. A prospective randomized trial of autologous bone marrow transplantation and chemotherapy for multiple myeloma. Intergroupe Français du Myelome. N Engl J Med 1996;335:91-7.

24 Lenhoff S, Hjorth M, Holmberg E, Turesson I, et al. Impact on survival of high-dose therapy with autologous stem cell support in patients younger than 60 years with newly diagnosed multiple myeloma: a population-based study. Nordic Myeloma Study Group. Blood 2000;95:7-11.

25 Lokhorst HM, Schattenberg A, Cornelissen JJ, Thomas LL, Verdonck LF. Donor leukocyte infusions are effective in relapsed multiple myeloma after allogeneic bone marrow transplantation. Blood 1997;90: 4206-11.

26 Gahrton G, Svensson H, Cavo M, Apperly J, et al. Progress in allogeneic bone marrow and peripheral blood stem cell transplantation for multiple myeloma: a comparison between transplants performed 1983-93 and 1994-8 at European Group for Blood 
and Marrow Transplantation centres. $\mathrm{Br} J$ Haematol 2001;113:209-16.

27 Alexanian R, Barlogie B, Dixon D. Highdose glucocorticoid treatment of resistant myeloma. Ann Intern Med 1986;105:8-11.

28 Singhal S, Mehta J, Desikan R, Ayers D, et al. Antitumor activity of thalidomide in refractory myeloma. $N$ Engl J Med 1999;341:1565-71.

Address for correspondence:

Professor Charles R J Singer, Consultant Haematologist,

Royal United Hospital, Combe Park, Bath BA1 3NG

\section{CME General Internal Medicine}

\section{for the Physician SAQs}

\section{Answers to the CME SAQs published in Clinical Medicine July/August 2001}

$\begin{array}{llllllllll}\text { Q1 } & \text { Q2 } & \text { Q3 } & \text { Q4 } & \text { Q5 } & \text { Q11 } & \text { Q12 } & \text { Q13 } & \text { Q14 } & \text { Q15 } \\ \text { a) } T & \text { a) } F & \text { a) } F & \text { a) } F & \text { a) } T & \text { a) } F & \text { a) } F & \text { a) } F & \text { a) } F & \text { a) } F \\ \text { b) } F & \text { b) } T & \text { b) } T & \text { b) } T & \text { b) } F & \text { b) } T & \text { b) } T & \text { b) } T & \text { b) } F & \text { b) } T \\ \text { c) } F & \text { c) } F & \text { c) } F & \text { c) } T & \text { c) } T & \text { c) } F & \text { c) } T & \text { c) } T & \text { c) } T & \text { c) } T \\ \text { d) } T & \text { d) } F & \text { d) } F & \text { d) } F & \text { d) } F & \text { d) } T & \text { d) } T & \text { d) } T & \text { d) } F & \text { d) } F \\ \text { e) } T & \text { e) } F & \text { e) } T & \text { e) } T & \text { e) } F & \text { e) } F & \text { e) } F & \text { e) } F & \text { e) } T & \text { e) } T \\ & & & & & & & & \\ \text { Q6 } & \text { Q7 } & \text { Q8 } & \text { Q9 } & \text { Q10 } & \text { Q16 } & \text { Q17 } & \text { Q18 } & \text { Q19 } & \text { Q20 } \\ \text { a) } T & \text { a) } T & \text { a) } F & \text { a) } F & \text { a) } F & \text { a) } T & \text { a) } T & \text { a) } F & \text { a) } T & \text { a) } F \\ \text { b) } F & \text { b) } F & \text { b) } F & \text { b) } F & \text { b) } F & \text { b) } T & \text { b) } F & \text { b) } F & \text { b) } F & \text { b) } F \\ \text { c) } T & \text { c) } T & \text { c) } F & \text { c) } F & \text { c) } T & \text { c) } F & \text { c) } F & \text { c) } F & \text { c) } T & \text { c) } F \\ \text { d) } T & \text { d) } F & \text { d) } T & \text { d) } T & \text { d) } F & \text { d) } F & \text { d) } T & \text { d) } F & \text { d) } F & \text { d) } T \\ \text { e) } T & \text { e) } T & \text { e) } T & \text { e) } T & \text { e) } F & \text { e) } T & \text { e) } T & \text { e) } T & \text { e) } F & \text { e) } T\end{array}$

